\title{
Optimization of the selection of sections in the distribution line considering reactive power compensation
}

\author{
Ivan Bandurin ${ }^{1 *}$, Vladimir Ivanov ${ }^{1}$, Igor Kozyrev ${ }^{1}$, Vladimir Korobov ${ }^{1}$, Alexey Khaimin ${ }^{1}$, Anastasia Martirosian ${ }^{1}$, Sergey \\ Trashchenkov ${ }^{1}$. \\ ${ }^{1}$ Pskov State University, Institute of Engineering Sciences, 180000, Russia, Pskov, Lenin Square, 2
}

\begin{abstract}
Today, the increase in reactive power consumption far exceeds the increase in active power consumption. Due to the increasing demands of the end-users for the quality of the supply of electricity, the problem of joint selection of rational sections and places of installation of reactive power compensation in the distribution line becomes relevant. A mathematical model and algorithm allowing such a choice are proposed. The mathematical model can be used both in the design of new lines and in the reconstruction of existing lines. An example is given.
\end{abstract}

\section{Introduction}

The problem of reactive power compensation in electrical networks is multifaceted and includes a number of subtasks, such as the choice of compensation devices, the choice of cross-sections of wires and cables, the improvement of the electrical quality indicators, the optimization of the load of transformers, Reduction of electricity costs and many others [1]. Numerous scientific studies have been devoted to these sub-tasks, both by the Electricity Network Organization and by consumers for different purposes (especially for industrial networks).

The work of [2] focuses on the problem of disconnect in the actions of electric network organizations and consumers in the installation of compensating devices. The most important influence on the reactive power balance is the installation of compensating devices near the loads, i.e. in the consumer networks, but the positive effect of such an installation also extends to the network organization. The consumer's lack of information about reactive power in the networks can lead to a bias. The authors [2] of propose a model and methodology for obtaining information on the possible reduction of network losses due to the installation of compensating devices on the side of consumers.

Publication [3] considers an example of the simplest electrical network, modelled in Rastrwin. In the case of a reactive load which varies during the day, the cases of a continuously operating compensating device, compensating device, disconnected during the night hours, compensating device, adjusted after load changes are considered. For all cases, a daily reduction of electricity losses is defined, and the role of regulation in reducing losses is shown.

Article [4] describes the use of FACTS (Flexible AC Transmission System) technologies such as static thyristor compensators and synchronous reactive power compensators to compensate for reactive power. On the basis of the description of technologies, analysis of world experience, it is concluded that this direction is promising. The present work explores ways of optimizing the crosssection of the wire and determining the locations of the compensating devices for new and reconstructed distribution lines. For this purpose, mathematical models of lines under different optimization conditions are presented.

\section{Mathematical model of optimization of section selection for line branch}

The result of the optimization task is to define the section for the branch line. The line section is selected according to the maximum possible load.

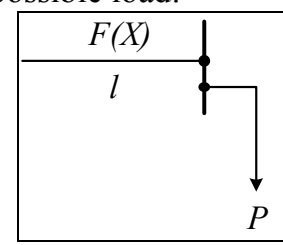

Fig. 1. Principle diagram for branch line.

The basic data are:

1) $l$ - is the length of wire, $m$;

2) $P$ - is the active power of the consumer, $\mathrm{kW}$;

3) $\cos \varphi$ - is the consumer's power ratio;

4) $U_{N O M}$ - is the nominal voltage of the consumer $\mathrm{kV}$;

5) $N_{P H A S E S}$ - is the number of phases of the consumer.

\section{Variable:}

$X$ - is an integer variable of line cross-section type.

$$
X \in[0,1 \mathrm{~K} m] \text {, }
$$

where $m$ is the number of cross-sectional types.

\section{Functions of:}

* Corresponding author: bandurin_ivan@mail.ru 
1) $F(X)$ - is the nominal cross-section of the wire, $\mathrm{mm}^{2}$;

2) $R_{S A R}(X)$ - is the specific active conductor resistance of wire, $\mathrm{Om} / \mathrm{km}$

3) $X_{S I R}(X)$ - is the specific inductive linear resistance of the wire, $\mathrm{Om} / \mathrm{km}$;

4) $I_{P C}(X)$ - is the permissible load current for wires, A;

5) $\quad C(X)$ - is the specific capital cost function of $1 \mathrm{~m}$ of line construction, dollar/m;

6) $I$ - value of the nominal current in the line, A.

To calculate the value of the nominal current in the line, the formula for a three-phase or single-phase current is used, known from electrical engineering.

7) $\Delta U(\mathrm{X})$ is the calculated voltage drop value of a line branch, V. Formula:

$$
\Delta U(X)=\frac{P \cdot R_{S A R}(X) l+Q \cdot X_{S I R}(X) l}{U_{N O M}},
$$

where $Q$ is the reactive power of the consumer, $\mathrm{kVar}$. We find her by the formula

$$
Q=\operatorname{Ptg} \varphi .
$$

\section{The objective function}

According to the [5] method, net discounted income may serve as the criterion for choosing the optimal crosssection of the wire. [6] shows that in many cases (for example, when the amount and price of electricity remain the same for all options) the criterion can be converted to a simpler minimum of reduced annual costs.

Minimum annual wire cost quoted

$$
\begin{aligned}
& O F=\left(E+\alpha_{A M}\right)(C(X) \cdot l)+ \\
& +\left(R_{\mathrm{SAR}}(X) \cdot l \cdot I^{2}\right) \cdot T_{\text {nhloss }} \cdot C_{E C} \rightarrow \mathrm{min} .
\end{aligned}
$$

where $E$ - is the discount rate; $\alpha_{\mathrm{AM}}$ - is the amortization factor; $T_{\text {nhloss }}$ - is the number of hours of the most losses of the line per year, $\mathrm{h} ; C_{E C}$ - is the electricity cost, $\mathrm{rub} / \mathrm{kW} * \mathrm{~h}$.

\section{Limitations:}

1) By variable type:

$X$ is an integer.

2) Over a range of variables:

$$
0 \leq X \leq m \text {. }
$$

3) By the minimum permitted ratio:

$$
F(X) \geq F_{M I N} \text {. }
$$

where $F_{M I N}$ - is the maximum section selected from the minimum allowable sections for various conditions (mechanical strength, holodeck, crown, etc.).

4) For long-term allowable continuous current

$$
I_{P E R M}(X) \geq I \text {. }
$$

5) For acceptable voltage loss

$$
\triangle U(X) \leq \Delta U_{P E R M} \text {. }
$$

Mathematical model 1 is the basis for subsequent mathematical models.

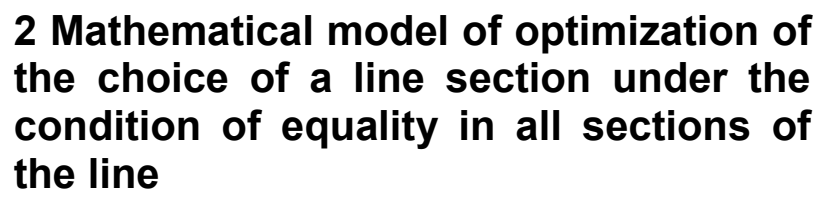

Selection of the section from the condition of equality on all sections of the line, $F_{j}=$ const $=F$. This condition is used to select the sections of wires and cables in urban electrical networks. The equality of the sections of wires provides the most advantageous conditions for the construction and installation of the network or its sections. This structure has special advantages for lines with a large number of loads, which are sufficiently close to each other [7].

As a result of the optimization problem, it is necessary to determine the value of one section for the main section of the line.

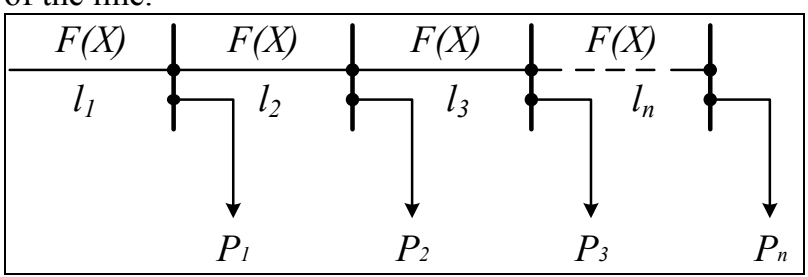

Fig. 2. Principle diagram of the projected line.

\section{Functions of:}

1) $\triangle U_{P E R M R}$ - is the calculated total voltage drop on the reactances in the line, $\mathrm{V}$;

Formula:

$$
\Delta U_{\text {PERM R }}=\frac{\sum_{i=1}^{n} \sum_{j=i}^{n} Q_{j} x_{0} l_{i}}{U_{N O M}} ;
$$

2) $\triangle U_{P E R M A}$ - is the calculated total voltage drop on active resistance in the line, $\mathrm{V}$;

Formula:

$$
\Delta U_{P E R M \mathrm{~A}}=\Delta U_{P E R M}-\Delta U_{P E R M R} ;
$$

3) $F_{C A L C}$ is the calculated value of the minimum section for the line, $\mathrm{mm}^{2}$. Formulae for the case of a threephase and a one-phase line:

$$
F_{C A L C}=\frac{\sum_{i=1}^{n} \sqrt{3} I_{i} l_{i} \cos \varphi_{i}}{\gamma \Delta U_{\text {PERM } A}} \text { or } F_{C A L C}=\frac{\sum_{i=1}^{n} I_{i} l_{i} \cos \varphi_{i}}{\gamma \Delta U_{\text {PERM } A}} ;
$$

4) $\Delta U_{M D}(X)$ is the calculated value of the largest voltage drop in the line, V. Formula:

$\Delta U_{M D}(X)=\sum_{i=1}^{n} \frac{\sum_{j=i}^{n} P_{j} \cdot R_{S A R}(X) \cdot l_{i}+\sum_{j=i}^{n} Q_{j} \cdot X_{S I R}(X) \cdot l_{i}}{U_{N O M}}$.

The objective function is:

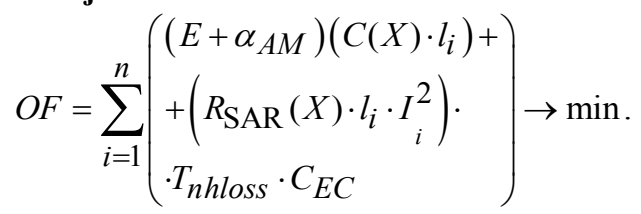

Limitations. The difference is that the cross-section of the wire in the line should not only be greater than the minimum permissible ratio, but should also be greater than the calculated ratio:

$$
F(X) \geq \min \left(F_{C A L C}, F_{M I N}\right) .
$$




\section{Mathematical model of optimization the selection of line sections under the minimum power loss condition}

It can be shown that the minimum power loss corresponds to a constant current density, i.e. the current density is the same for all sections of the line. This additional condition is used in the choice of sections in the grid of electrical power supply systems of industrial enterprises. In these networks, relatively short lines and high loads, i.e. the metal consumption is low, and power losses are high [7].

As a result of the optimization task it is necessary to define all sections for the main section of the line.

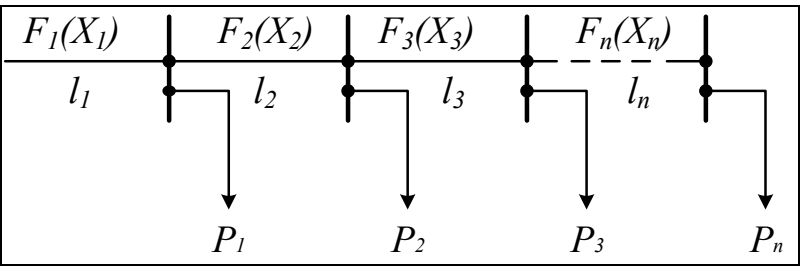

Fig. 3 Principle diagram of the projected line.

\section{Variables:}

$X_{1}, X_{2} \ldots X_{n}$ - is an integer variable of the type of the cross section of the line.

Functions of:

1) $J_{\Delta U}$ - is the calculated current density, $\mathrm{A} / \mathrm{mm}^{2}$;

Formula for the case of a three-phase or one-phase line:

$$
J_{\Delta U}=\frac{\gamma \Delta U_{\text {PERM A }}}{\sqrt{3} \sum_{i=1}^{n} l_{i} \cos \varphi_{i}} \text { or } J_{\Delta U}=\frac{\gamma \Delta U_{\text {PERM } A}}{\sum_{i=1}^{n} l_{i} \cos \varphi_{i}} .
$$

2) $F_{C A L C i}-$ is the calculated section on the i-section of the motorway, $\mathrm{mm}^{2}$;

Formula:

$$
F_{C A L C i}=\frac{I_{i}}{J_{\Delta U}} .
$$

3) $\Delta U_{M D}(X)$ - is the calculated value of the largest voltage drop in the line, $\mathrm{V}$;

Formula:

$$
\begin{aligned}
& \Delta U_{M D}\left(X_{1}, X_{2} \mathrm{~K} X_{n}\right)= \\
& =\sum_{i=1}^{n} \frac{\sum_{j=i}^{n} P_{j} \cdot R_{S A R}\left(X_{i}\right) \cdot l_{i}+\sum_{j=i}^{n} Q_{j} \cdot X_{S I R}\left(X_{i}\right) \cdot l_{i}}{U_{N O M}} .
\end{aligned}
$$

The objective function is:

$$
O F=\sum_{i=1}^{n}\left(\begin{array}{l}
\left(E+\alpha_{A M}\right)\left(C\left(X_{i}\right) \cdot l_{i}\right)+ \\
+\left(R_{S A R}\left(X_{i}\right) \cdot l_{i} \cdot I_{i}^{2}\right) . \\
\cdot T_{\text {nhloss }} \cdot C_{E C}
\end{array}\right) \rightarrow \min .
$$

Limitations:

1) Variable type

$$
X_{1}, X_{2} \ldots X_{n} \text { - is an integer. }
$$

2) Over a range of variables:

$$
\left\{\begin{array}{l}
0 \leq X_{1} \leq m \\
0 \leq X_{2} \leq m \\
\mathrm{~L} \\
0 \leq X_{n} \leq m
\end{array}\right.
$$

3) For long-term allowable continuous current

$$
\left\{\begin{array}{l}
I_{\text {PERM }}\left(X_{1}\right) \geq I_{1} ; \\
I_{\text {PERM }}\left(X_{2}\right) \geq I_{2} ; \\
\mathrm{L} \\
I_{\text {PERM }}\left(X_{n}\right) \geq I_{n} .
\end{array}\right.
$$

4) By the minimum permitted ratio:

$$
\left\{\begin{array}{l}
F_{1}\left(X_{1}\right) \geq F_{C A L C 1} ; \\
F_{2}\left(X_{2}\right) \geq F_{C A L C 2} \\
\mathrm{~L} \\
F_{n}\left(X_{n}\right) \geq F_{C A L C n} .
\end{array}\right.
$$

5) According to permissible voltage loss

$$
\Delta U_{M D}\left(X_{1}, X_{2} \mathrm{~K} X_{n}\right) \leq \Delta U_{P E R M} .
$$

\section{Mathematical model of optimization the selection of line section of line sections from the minimum flow of conductor material into the construction of the line}

This condition is used in rural networks at low load, where saving metal is more important than saving electricity losses [7].

Here, as in the previous problem, we need to define all sections for the main section of the line.

\section{Functions of:}

1) $F_{C A L C n}$ - is the calculated section on the n-section of the motorway, $\mathrm{mm}^{2}$;

Formula:

$$
F_{C A L C n}=\frac{\rho \sqrt{P_{n}}}{\Delta U_{P E R M A} \Delta U_{N O M}} \sum_{j=1}^{n} l_{j} \sqrt{P_{j}},
$$

2) $F_{C A L C n-1}-$ is the calculated section on the n- 1 section of the motorway, $\mathrm{mm}^{2}$;

Formula:

$$
F_{C A L C n-1}=\sqrt{\frac{F_{C A L C n}^{2} P_{n-1}}{P_{n}}} .
$$

\section{Mathematical model of optimization of capacitor in the distribution line}

The activation of the compensation devices in the vicinity of the receivers consuming the reactive power results in the unloading of the network elements. The use of compensating devices makes it possible to improve the quality of electricity, reduce losses and increase the capacity of the distribution line.

In the mathematical model, we expect different types of capacitor batteries of standard power to be installed. As a result of the optimization task, it is necessary to 
determine the number and type of capacitor batteries installed, as well as the locations of their installations.

\section{Variable:}

The $n_{i j}$ - integer variable number of $i$-type capacitors installed at the $j$-th place.

The objective function, considering the stated costs, is generally as follows:

$$
\begin{aligned}
& O F=\sum_{i=1}^{n} \sum_{j=1}^{l} n_{C D i j}\left(E+\alpha_{A M}\right) C_{C D i}+ \\
& +\sum_{k=1}^{m} a_{L}\left(P_{L k}^{2}+\left(Q_{L k}-\sum_{i=1}^{n} \sum_{j=1}^{l} n_{C D i j} Q_{C D i}\right)^{2}\right) \rightarrow \\
& \rightarrow \text { min, }
\end{aligned}
$$

where the combined active and reactive power of the line

$$
P_{L k}=\sum_{j=1}^{m} P_{L O A D j}, Q_{L k}=\sum_{j=1}^{m} Q_{L O A D j} ;
$$

$Q_{C D}$ is the power of the compensating device to be installed;

$C_{C . C O S T}$ - unit capital costs of compensating devices and switching equipment;

$a_{L}$ - is the ratio that considers resistance, the time of greatest loss, and the cost of energy losses at the tariff, in the general case the coefficient

$$
a_{L}=\frac{R_{L} c_{0} T_{\ni}}{U_{\text {NOM }}^{2}} .
$$

\section{Limitations:}

1) The variable numbers of capacitor batteries installed shall be integer:

$$
n_{C D i j}-\text { is an integer. }
$$

2) The variable numbers of capacitor batteries installed shall be greater than or equal to zero:

$$
n_{C D i j} \geq 0 \text {. }
$$

3) The voltage deviation at network nodes shall be between -0.1 and 0.1 :

$$
\left|\frac{\Delta U_{j}}{U_{N O M}}\right| \leq 0.1,
$$

where the power drop in the line

$$
\Delta U_{j}=\sum_{k=1}^{m} \frac{P_{L k} R_{L k}+\left(Q_{L k}-\sum_{i=1}^{n} \sum_{j=1}^{l} n_{C D i j} Q_{C D i}\right) X_{L k}}{U_{N O M}} .
$$

4) The current in the sections of the distribution line shall not exceed the maximum allowable current for the conductor.

$$
I_{\text {CALC } k} \leq I_{\text {PERM } k},
$$

where the calculated current is in the $k$-th section of the line

$$
I_{C A L C k}=\frac{\sqrt{P_{L k}^{2}+\left(Q_{L k}-\sum_{i=1}^{n} \sum_{j=1}^{l} n_{C D i j} Q_{C D i}\right)^{2}}}{\sqrt{3} U_{N O M}} .
$$

To illustrate the mathematical model, the simplest model of the distribution line (figure 4) was selected.

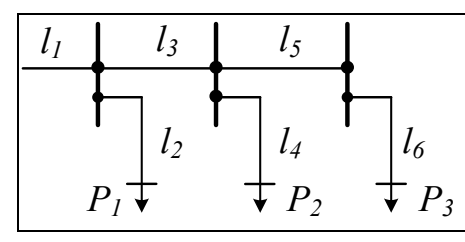

Fig. 3 Basic diagram of the simplest distribution line for

Variables: example.

$n_{C D 11}, n_{C D 12}, n_{C D 13}, n_{C D 21}, n_{C D 22}, n_{C D 23}-$ is an integer variable number of $i$-type capacitors installed in $j$ place.

The objective function, considering the stated costs, is generally as follows:

$$
\begin{aligned}
& O F=\sum_{i=1}^{2} \sum_{j=1}^{3} n_{C D i j}\left(E+\alpha_{A M}\right) C_{C D i}+ \\
& +\sum_{k=1}^{6} a_{L}\left(P_{L k}^{2}+\left(Q_{L k}-\sum_{i=1}^{2} \sum_{j=1}^{3} n_{C D i j} Q_{C D i}\right)^{2}\right) .
\end{aligned}
$$

Limitations:

1) The variable numbers of capacitor batteries installed shall be integer:

$$
\begin{aligned}
& n_{C D 11}, n_{C D 12}, n_{C D 13}, \\
& n_{C D 21}, n_{C D 22}, n_{C D 23}-\text { is an integer. }
\end{aligned}
$$

2) The variable number of capacitor batteries installed shall be greater than zero:

$$
n_{C D 11}, n_{C D 12}, n_{C D 13}, n_{C D 21}, n_{C D 22}, n_{C D 23} \geq 0 \text {. }
$$

3) The voltage deviation at network nodes shall be between -0.1 and 0.1 :

$$
\left|\frac{\Delta U_{j}}{U_{N O M}}\right| \leq 0.1 .
$$

Below is an expression for calculating the voltage drop from the start of the line to the first user

$$
\begin{gathered}
P_{L 1} R_{L 1}+\left(\begin{array}{l}
Q_{L 1}-\left(n_{C D 11} Q_{C D 1}+\right. \\
+n_{C D 21} Q_{C D 2}+n_{C D 12} Q_{C D 1}+ \\
+n_{C D 22} Q_{K Y 2}+n_{C D 13} Q_{C D 1}+ \\
\left.+n_{C D 23} Q_{C D 2}\right)
\end{array}\right) X_{L 1} \\
U_{N O M}=\frac{P_{L 2} R_{L 2}+\left(Q_{L 2}-\left(n_{C D 11} Q_{C D 1}+n_{C D 21} Q_{C D 2}\right)\right) X_{L 2}}{U_{N O M}} .
\end{gathered}
$$

For other users, the voltage reduction formula is calculated by analogy.

4) Current in the lines shall not exceed the maximum permissible current for this conductor.

$$
I_{\text {CALC } k} \leq I_{\text {PERM } k} \text {. }
$$

As an example, the expression below is for the calculated current on the 1 st section of the line 


$$
I_{C A L C L 1}=\frac{\sqrt{P_{L 1}^{2}+\left(\begin{array}{l}
Q_{L 1}-\left(n_{C D I 1} C_{C D I 1} Q_{C D 1}+\right)^{2} \\
+n_{C D 21} C_{C D 21} Q_{C D 2}+ \\
+n_{C D 12} C_{C D 12} Q_{C D 1}+ \\
+n_{C D 22} C_{C D 22} Q_{C D 2}+ \\
+n_{C D 13} C_{C D 13} Q_{C D 1}+ \\
\left.+n_{C D 23} C_{C D 23} Q_{C D 2}\right)
\end{array}\right)^{2}}}{\sqrt{3} U_{N O M}} .
$$

\section{Mathematical model of optimization of line section selection with compensations for reactive power in consumers}

This mathematical model is a combination of the mathematical models discussed above. As a result of the optimization task, it is necessary to determine the sections in the distribution line and the advisability of installing capacitor batteries in consumers.

The mathematical models that we have considered belong to the class of integer non-linear programming problems. To find an exact or approximate solution, use the appropriate known methods of solving such problems [8]. When using a PC to obtain a solution to a non-linear optimization problem, it is convenient to use Microsoft Excel [9]. The resulting solution is analyzed and the optimal sections in the line are selected.

\section{Conclusion}

Mathematical models and algorithms for selecting wire sections in a line under different optimization conditions have been improved. The problem of joint selection of rational sections and locations of the reactive power compensation unit in the distribution line is solved. Mathematical models can be used both in the design of new lines and in the reconstruction of existing lines.

Further research should focus on the development of mathematical models for the selection of wire sections in a line with dynamic compensation of reactive power.

\section{References}

1. Y.S. Zhelezko, Calculation, Analysis and Rationing of Electricity Losses in Electrical Networks. Manual for practical calculations / U.S. Zhelezko, A.V. Artemiev, O.V. Savchenko. - M.: CENAS, (2006), 280 p.

2. A.V. Kuznetsov, I.V. Argentova, Mathematical model of estimation of reduction of power losses in network organization at compensation of reactive power //Electrotechnics, 10 (2016), p. 68-73.

3. N.S. Ponyavin, E.A. Yerkov, Assessment of the influence of reactive power compensation regulation on the operating modes of the electrical network //Youth Vector for the Development of Agricultural Science. (2018), p. 136-141.

4. N.N. Solonina, K.V. Suslov, Z.V. Solonina, New Reactive Power Compensation Technologies /Bulletin of the Irkutsk State Technical University, 5(112), (2016), p. 135-143.

5. Guidelines for evaluating the effectiveness of investment projects and their selection for financing (second revision). Official publication, (2000), $421 \mathrm{pp}$. 6. V.G. Kitushin, Reliability of energy systems. C. 1. Theoretical framework : [Manual] / V. G. Kitushin. Novosibirsk, (2003), p. 135-143.

7. V.I. Idelczyk, Electrical systems and networks. - M.: Energocom Publishes (1989), 592 pp.

8. Wikipedia: site. - (2020) - URL: https:/ru/ru.wikipedia.org/wiki/CEL_programming\#Algo rithms/ (Date of Referral: 19.09.2020).

9. V.N. Kostin, Optimization of the electric power industry: Training. manual. - Spb.: NWT, (2003), 120 p. 